\title{
Lesson of the month 2: Contusion confusion
}

\author{
Authors: Helen Wood, ${ }^{\mathrm{A}}$ Thomas Mayes, ${ }^{\mathrm{B}}$ Susan King, ${ }^{\mathrm{C}}$ Joanna Kasznia-Brown ${ }^{\mathrm{C}}$ and Tarun Solanki ${ }^{\mathrm{D}}$
}

This lesson of the month highlights that certain radiology terminology may be used to report bleeding on head computerised tomography (CT) reports. On-call junior doctors should not be expected to interpret CT head images, so often their decisions will be based on the written report. The wording used can change the clinical decision and therefore the treatment given by a junior doctor. Clinical teams and junior doctors should be educated on terminology in relation to bleeding on CT head reports.

KEYWORDS: Acute intracranial haemorrhage, anticoagulation, CT head, contusion, fall

\section{Case history}

A 77-year-old female was admitted to hospital with acute confusion and urinary sepsis. Prior to admission she was independent - undertaking activities of daily living, managing short distances with a stick and using an electric scooter outdoors. Notably, the patient was on warfarin for atrial fibrillation.

During her inpatient stay - in the early hours of the morning she fell, sustaining a head injury. A normal neurological examination was documented within 1 hour of the fall and a computerised tomography (CT) head was arranged because of head trauma in the context of a patient on an anticoagulant.

The following is the CT head report as per the on-call consultant radiologist:

There is some patchy foci of high attenuation in the right frontal cortex. Tiny focus also left frontal lobe and right parietal lobe. Mild low attenuation in the deep white matter, likely small vessel ischaemia. High attenuation round structure, $11 \mathrm{~mm}$ over the convexity sulci.

Left parietal lobe. No skull fracture seen.

\section{IMPRESSION:}

Likely small cerebral contusions as described.

Likely small left parietal convexity sulcal meningioma.

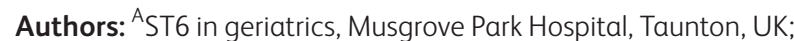
${ }^{B}$ ST5 in geriatrics, Musgrove Park Hospital, Taunton, UK; ${ }^{C}$ consultant radiologist, Musgrove Park Hospital, Taunton, UK; ${ }^{D}$ consultant geriatrician, Musgrove Park Hospital, Taunton, UK

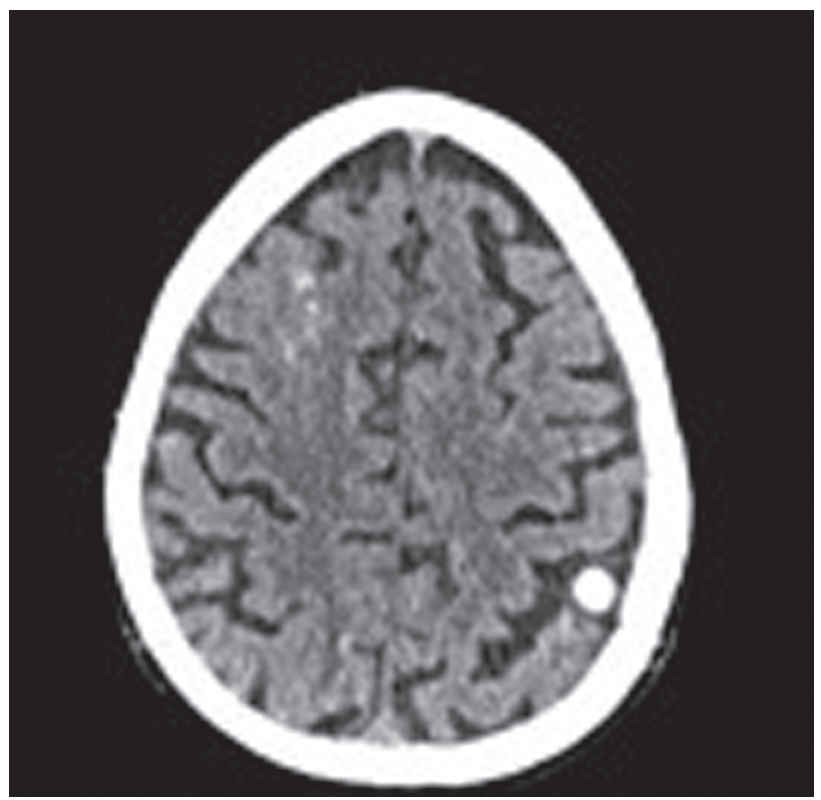

Fig 1. Initial computerised tomography head scan.

Based on the above report, the on-call junior doctor continued neurological observations but did not reverse warfarin nor discuss the report with the neurosurgeons.

Later that day, the patient developed sudden onset of speech disturbance, left-sided facial weakness, left gaze palsy and a left upper and lower limb hemiparesis. Her Glasgow Coma Score (GCS) was 12/15.

An urgent repeat CT head was arranged. Her international normalised ratio at this point was 1.8

The repeat CT head showed a subdural haematoma with mass effect and midline shift. There was a small subarachnoid haemorrhage. Neurosurgical referral was advised.

GCS decreased to 6/15 within 2 hours of the initial neurological signs.

Her coagulopathy was reversed with Octaplex and vitamin K. The on-call junior doctor discussed the case with the tertiary neurosurgical unit and because of the patient's history of sepsis and comorbidities, a conservative approach was recommended. The patient's GCS continued to decline and she developed an aspiration pneumonia from which she subsequently died.

The patient's death was referred to the coroner and an inquest was held. 


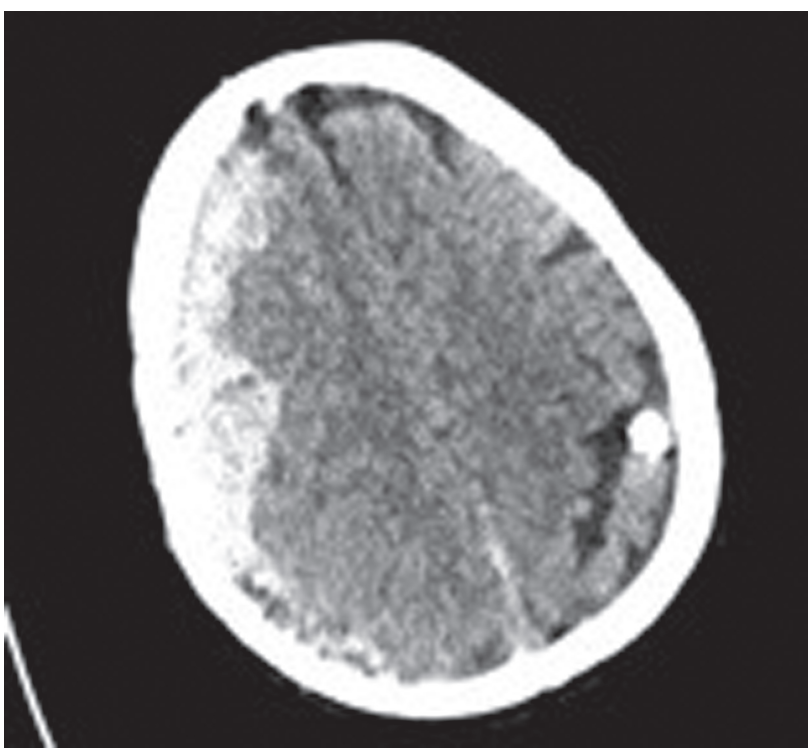

Fig 2. Second computerised tomography head scan.

\section{Discussion and the coroner's inquest}

On-call juniors should not be expected to interpret CT head images and often their decisions will be based on the CT head report. The decision not to reverse warfarin was initially made on the basis that the word 'contusion' was used in the report and not 'haemorrhage' or 'bleed'. This was discussed at our departmental radiology meeting and the images were reviewed with a consultant radiologist. The original scan did show evidence of fresh bleeding around the contusion site, which could have been reported as haemorrhage.

We asked colleagues both junior and senior, given the initial CT head report, whether they would they have reversed the warfarin. The results were mixed, showing confusion around the use of the word 'contusion' in the report. Had the report used the word 'haemorrhage', everyone agreed that they would have reversed the warfarin.

The coroner's inquest reported that the junior doctor reviewing the CT head report did not recognise it 'as demonstrating evidence of an intracerebral haemorrhage' and hence did not reverse her warfarin. Had this been done, the risk of the subsequent fatal subdural haematoma would have been reduced.
The case was discussed locally in our radiology multidisciplinary team meeting and it was further reviewed in the radiology departmental meeting. The radiology department state it is normal practice for radiologists to report such appearances as seen on the first CT head following head injury as "contusion", as they represent bruising in the brain cortex and parenchyma. "Intracerebral bleed" is usually used in relation to subdural or extradural haematoma, subarachnoid haemorrhage or large intraparenchymal bleed'.

At the radiology departmental meeting, all radiologists present reviewed the scans without accessing the official report. Everyone agreed that an acute contusion was present in keeping with the original report. It was agreed that clinical teams and junior doctors should be educated on terminology in relation to bleeding on CT head reports. However, the radiologists recognised that junior doctors have limited clinical experience and work under extreme time pressures. Therefore, to improve patient safety, it was agreed to produce a well-structured and clear conclusion on reports to ensure communication of acute conditions. It was agreed that clear phrases, such as haemorrhagic contusions or intracranial haemorrhage, should be used in such reports. If there is no acute bleed, the conclusion should state 'NO evidence of acute intracranial haemorrhage'.

These changes will improve patient care by preventing similar misinterpretations of radiology reports in the future. We will provide education to clinicians so that they understand the significance of a 'contusion' on head CT reports. We encourage all hospitals to review their radiology reporting terminology around contusions, intracerebral bleeds and their reporting protocols to ensure a clear conclusion.

\section{Conflicts of interest}

The authors have no conflicts of interest to declare.

\section{Acknowledgements}

Consent for publication was obtained from the patient's family.

Address for correspondence: Dr Helen Wood, Musgrove Park Hospital, Parkfield Drive, Taunton TA1 5DA, UK.

Email: helenwood@doctors.org.uk 